AperTO - Archivio Istituzionale Open Access dell'Università di Torino

\title{
Estimation of the workload in horses during an eventing competition
}

\section{This is the author's manuscript}

Original Citation:

Availability:

This version is available http://hdl.handle.net/2318/138371

since 2022-01-26T17:29:53Z

Terms of use:

Open Access

Anyone can freely access the full text of works made available as "Open Access". Works made available under a Creative Commons license can be used according to the terms and conditions of said license. Use of all other works requires consent of the right holder (author or publisher) if not exempted from copyright protection by the applicable law. 


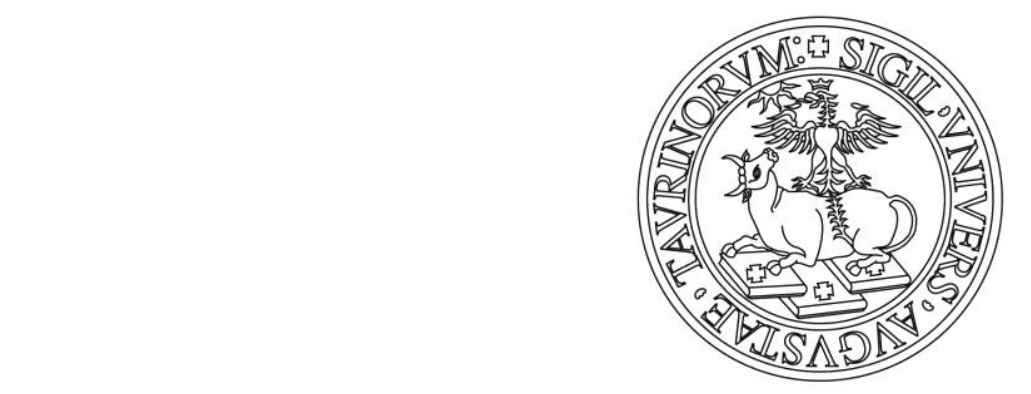

\section{UNIVERSITÀ DEGLI STUDI DI TORINO}

This is an author version of the contribution published on:

Questa è la versione dell'autore dell'opera:

[Estimation of the workload in horses during an eventing competition

Comparative Exercise Physiology,20139 9, 93-102]

10.3920/CEP12028

The definitive version is available at:

La versione definitiva è disponibile alla URL:

https://wageningenacademic.metapress.com/content/62k2862257471622/resource -secured/?target=fulltext.pdf 
Estimation of the workload in horses during an eventing competition

E. Valle1, R. Odore1, P., R. Zanattal, P. Badinol, C. Girardi1,J. Nery1, A. Assenza 2, and D.

Bergerol

1Department of Veterinary Sciences, University of Turin, Via L. Da Vinci 44, Grugliasco, Italy

2 Department of Experimental Sciences and Applied Biotechnology, University of Messina, Polo

Universitario Annunziata, 98168 Messina, Italy.

\begin{abstract}
The aim of this study was to evaluate workload using suitable parameters related to the physical effort exerted by horses involved in eventing competitions in order to describe the workload intensity and energy demands placed upon such horses. Heart rate (HR), running speed (S), distance covered (D) and blood lactate (Lact) concentrations were measured in horses competing at either the intermediate level (IL) or advanced level (AL) in order to identify workload differences between experience class.

Ten warmblood horses were monitored during an official two-day eventing competition; mean HR (HRmean), maximum HR (HRmax), mean speed (Smean, $\mathrm{m} / \mathrm{min}$ ), max speed (Smax, $\mathrm{m} / \mathrm{min}$ ) and performance duration (D, min) were assessed during the warm-up (WU) and competition (C) phases of each eventing test (dressage, show jumping, cross country). To calculate heart rate recovery (HRR), HR data were collected within the first 3 minutes following the completion of each of the 3 competition phases. Energy expenditure (EE) was estimated using the HR/VO2 relationship. Differences between the groups (AL vs. IL) in HRmean, HRmax, \%HRmean, \%HR peak (HR expressed as a percentage of the maximum HR peak obtained during a fast gallop training session), $\mathrm{S}$, and $\mathrm{D}$ were assessed using one-tailed unpaired $t$-tests for both WU and $\mathrm{C}$ phases; the relationship between HR and S was also determined for WU and C phases using one-tailed Person's correlations. The relationship between HR decrease during the first 3 minutes following competition phase completion and recovery time was investigated by multiple nonlinear curve estimation procedures.

The results reveal the cross country test to be the most demanding of the eventing competition, requiring significantly greater levels of muscular and energetic effort, in terms of Lact production and EE, with higher values recorded in the AL horses compared to IL horses. The data also show that riders need to optimise WU duration and quality in accordance with their competition category. The calculation of HRR is also shown to be an appropriate approach for gauging workload after high-intensity exercise, but not after low-intensity exercise since HRR may be influenced by external factors, like how excited a horse is.

Key words: horse, exercise physiology, dressage, show jumping, cross country Manuscript
\end{abstract}

\title{
1. Introduction
}

Eventing is recognised as one of the most challenging equestrian disciplines (Murray et al., 2006). Eventing combines three different tests in one competition: dressage (Dr), judged on the quality of gaits and accuracy of movements (Williams et al., 2009), show jumping ( $\mathrm{Sj}$ ), where ability skills are requested, and cross country $(\mathrm{Cr})$, which tests strength and bravery in both the horse and rider (Marlin, 2007).

The eventing format that has received the most attention within the scientific literature is the long format (LF). In this competition format, the dressage test takes place on the first day and the show jumping is held on the third day. The second day is dedicated to the cross country test, which includes four phases: phases A and C (roads and tracks), where the horse is required to cover a fixed distance with no jumping efforts involved; phase B, the steeplechase, covering a shorter distance and 5-10 steeplechase fences; and phase D, the cross country course, covering a distance of 3,900-7,980 $\mathrm{m}$ and a maximum of 45 jumps (Murray et al., 2006). 
The so-called short format (SF) of eventing competition also exists and has become increasingly popular over recent years; introduced in 2004 into prestigious competitions, such as the Athens Olympic Games (Murray et al 2006; Marlin, 2007). This format excludes phases A, B, and C from the endurance day; moreover, the cross country course covers a fixed distance and involves jumps that require more intense physical effort, rendering the test more spectacular. Furthermore, since the year 2000, changes have been introduced into the dressage test such that it now requires more skill: for example, the FEI (International Federation for Equestrian Sports) dressage tests at the 4-star level can be considered approximately equivalent to the Advanced Medium level in pure Dressage (Marlin, 2007).

Very few studies have addressed the physiological effects occurring in horses during SF eventing, despite, the fact that monitoring horses during competition can help optimise training programmes for eliciting adaptation responses in the athlete horse (Williams et al., 2009). Horses need to be carefully trained to improve strength capacity and cardiovascular efficiency (Clayton, 1991). An adequate fitness level is necessary, especially in the case of high level competitions, in order to avoid accidents that could represent a source of dispute from the welfare point of view.

The measurement of heart rate (HR) during exercise has been shown to be an objective tool for estimating workload in horses (Dekker et al., 2007). HR varies according to metabolic demands and can thus be considered a gauge of cardiovascular, metabolic, muscular and locomotor variables. For this reason, according to Coenen (2010), HR can be used as an index of workload. Moreover, it is easy to record using commercially available instruments under practical conditions, like during competitions. The same author has elaborated a suitable model for predicting energy expenditure during work using HR.

The most commonly used procedure for evaluating energy demands during physical activities is indirect calorimetry, based on the oxygen uptake (VO2) measurement; however, oxygen uptake is technically difficult to measure, requiring a mask being applied over the horse's nose, and most studies that have used this technique to investigate energy expenditure during exercise have done so using standardised exercise tests on high-speed treadmills. HR, on the other hand, serves as a reliable predictor of VO2 (Conen, 2010) and provides a more practical method for use in animal studies for assessing energy demands during locomotion (Reis et al., 2011). HR increases in direct proportion to running speed. However, a point occurs beyond which an increase in speed does not result in additional increases in HR. When this plateau phase is reached, then it may be assumed that it represents the highest HR value, usually ranging between 210 and 240 beats per minute in equine athletes (Evans et al., 2006). Although a plateau in HR is difficult to reach in the field, it is possible to record a maximal HR peak (HRpeak) during a fast gallop training session. According to Gramkow and Evans (2006), maximal HR values are reported to have a median of $215 \mathrm{bpm}$ in the thoroughbred.

Following exercise, the decrease in HR, i.e. heart rate recovery (HRR), is a useful parameter to measure since it is influenced by the degree of fitness. Indeed, the HR decrease is widely used to evaluate performance or fitness in endurance horses and it is even measured at competition veterinary gate examinations where a decrease in HR to $64 \mathrm{bpm}$ or less within a set time frame is required for a horse to continue in the competition.

Other important variables used to describe workload are running speed (S), distance covered (D) and lactate (Lact) production (Rogers et al., 2007). The collection of these data, not only provides important information to assess the degree of training required (Serrano et al., 2002), but it also aids the evaluation of work intensity and the energy demands required by specific disciplines.

Since studies in the scientific literature evaluating effort during eventing competitions, especially in short format events, are limited (Murray et al., 2006), the present study was performed to investigate the workload of trained horses during an official short format eventing competition; horses competing at either the intermediate level (IL) or advanced level (AL) category were investigated. The most commonly used parameters of workload are HR and blood lactate concentrations. In addition to these, the present study also evaluates performance duration (D), 
mean running speed (S), distance covered (D), and HRR during both the warm-up (WU) and competition (C) phases of each eventing test (dressage, show jumping, cross country) in order to investigate whether these indexes of workload are higher in horses that compete at the AL compared to the IL.

\section{Materials and methods}

\section{Animals}

Ten warmblood horses ( 7 geldings, 1 stallion and 2 females) were recruited onto the study (mean body weight: $579 \pm 37 \mathrm{Kg}$; mean body condition score, according to Henneke et al., 1983; 5.8 \pm 0.7 ). All the horses came from the same Eventing Stables and were housed in individual stalls with wood chip bedding. They received first cut meadow hay, composed essentially of Lolium Italicum L., and a commercial cereal mix feed (crude protein $12.5 \%$, crude fat $5.2 \%$, crude ash $8.5 \%$, crude fibre $8.5 \%$ ) provided in three daily meal in order to supply a total of $66.57 \pm 2.82 \mathrm{MJ} /$ day of net energy, $10.5 \pm 2.6 \mathrm{~kg} /$ day of dry matter and $697 \pm 160 \mathrm{~g} /$ day of MADC (Horse Digestible Crude Protein). The forage to concentrate ratio was 58:42. Horses were trained according to the same physical conditioning programme for the competition: flat work (50 min/day, 3 day/wk.), dressage work and jumping (50 min/day, 2 day/wk.) and galloping (warm-up and galloping at $450 \mathrm{~m} / \mathrm{min}$ for $5-8$ minutes once a week). All horses regularly participated in eventing competitions. Before the beginning of the study, all animals underwent a veterinary visit, including a cardiologic examination, respiratory auscultations and routine haematology and plasma biochemistry. Only clinically healthy animals were included in the study.

\section{Competition features}

Data were collected during an official two-day eventing competition performed in northern Italy in May 2009. The weather conditions were relatively stable throughout the two days of competition (mean temperature $19^{\circ} \mathrm{C}$, relative humidity $66 \%$ ). During the first day, horses completed the dressage and show jumping tests, and the cross country test was performed on the second day. All the tests were performed between 8:30-11:30 a.m. and the grass ground conditions were similar for all horses, independent of their starting order. The horses were ridden by their own riders; 5 horses competed at the IL and 5 horses at the AL, according to their qualifications and preparation. A detailed description of the competition features is reported in Table 1.

\section{Exercise test and competition features}

Two weeks before the competition, horses were tested on a track in a fast gallop training session. According to Gramkow and Evans (2006), it is possible to reach velocity at maximal heart rate in the field, without using a standardised exercise test: after a warm-up of 25 minutes, all horses were ridden by the same professional rider to the highest speed possible over $1200 \mathrm{~m}$ on a sand track in order to evaluate their maximum recordable HR (HRpeak).

\section{Data collection}

For the evaluation of HR, horses were equipped with a heart rate monitor and GPS system (Polar Equine RS800 G3 Polar Electro OY, Kempele, Finland). The belt containing the electrodes was placed under the saddle. The recording watch was set to record every 5 seconds. HR monitoring started at the beginning of the warm-up session and continued until the horse was returned to his box after the competition. The exact starting and finishing time of each WU and $\mathrm{C}$ phase was recorded for each test $(\mathrm{Dr}, \mathrm{Sj}, \mathrm{Cr})$ by students in order to correlate the recorded HR data to each horse's activity.

The GPS device used to record ground distances covered was fixed directly to the saddle to maximise satellite reception.

Eight to ten minutes following the completion of each test, blood samples were collected from the jugular vein for the immediate measurement of blood lactate concentrations using a portable blood lactate analyser (Lactate pro LT-1710, Arkay, Japan), as previously used for horses (Sloet van Oldruitenborgh-Oosterbaan et al., 2008). This time frame was conditioned by the official 
regulations of the event, which only allowed blood samples to be taken once a horse has returned to his box.

\section{Data manipulation}

HR and GPS data were elaborated using Polar Pro trainer 5 (Polar Electro OY, Kempele, Finland) equine edition software for the assessment of: mean heart rate (HRmean); maximum heart rate recorded (HRmax) - also expressed as a percentage of the peak HR obtained during the fast galloping test (\%HRmean) and maximum HR (\%HRmax); mean running speed (Smean, m/min); max running speed (Smax, $\mathrm{m} / \mathrm{min})$; and duration $(\mathrm{D}, \mathrm{min})$ for all $\mathrm{WU}$ and $\mathrm{C}$ performances. The heart rate data for the first three minutes following completion of each competition test performance were extracted from the cardiac output file for the calculation of heart rate recovery (HRR).

To assess energy expenditure (EE), an estimate of oxygen consumption (VO2; $\mathrm{mL} / \mathrm{kgBw} / \mathrm{min}$ ) was calculated using mean HR (bpm) and $\mathrm{S}(\mathrm{m} / \mathrm{s})$ data, according to the model developed by Coenen (2008) 2008 that elaborated a suitable model to predict oxygen consumption by heart rate and speed: VO2=0.001946HR2.0648 and VO2 $=4.515+9,14(\mathrm{~S})+0,726(\mathrm{~S}) 2-0,00452(\mathrm{~S}) 3$. Indirect calorimetry assumes that $1 \mathrm{ml}$ of oxygen is equivalent to $20.1 \mathrm{~J}$ of energy consumed. Thus, the calculated VO2 must be expressed as a measure of heat or as EE in joules, which can then be converted to Kcal or MJ (Coenen, 2008).

Statistical analysis

All data were analysed using SPSS $17.0 \circledR$ software. The level of significance was set at $P<0.05$. After checking for normality, data were analysed using one-tailed unpaired t-tests to compare HRmean, HRmax, \%HRmean, \%HRmax, S and D during WU and C performances between horses competing at $\mathrm{AL} v s$. IL for each test ( $\mathrm{Dr}, \mathrm{Sj}, \mathrm{Cr}$ ).

The relationship between HR and S for WU and C phases was determined by one-tailed Pearson's correlations. The relationship between HR decrease and recovery time was investigated using multiple nonlinear curve estimation procedures for each test. The model with the highest coefficient of determination (r2) was accepted as the optimal one for the relationship.

\section{Results}

\section{Heart rate}

The range of peak heart rates (HRpeak, bpm) recorded in the fast gallop training session are reported in Table 2, divided by the competition level at which the horses compete (AL vs. IL). Mean HR values for AL vs. IL horses recorded during the WU and C phases of each of the three tests are reported in Tables 3 and 4.

During the WU and $\mathrm{C}$ phases of the Dr test, the IL horses showed significantly higher \%HRmean $(\mathrm{P}=0.04)$ and higher \%HRmax $(\mathrm{P}=0.05)$ than AL horses (Table 4). In the Sj test, differences between AL vs. IL were only recorded in the WU phase, with a significantly higher \%HRmean in the horses competing at IL $(P=0.05)$. During the $\mathrm{Cr}$ test, although no significant differences between the two groups were recorded in the WU phase, a tendency was observed for AL horses to have higher \%HRmean during the $\mathrm{C}$ phase $(P=0.06)$ (Table 3 and 4$)$.

\section{Time, distance and running speed}

Table 5 reports the mean values for the time required to complete each phase (WU and C) of the three eventing tests, the total distance travelled and the average and maximum running speeds recorded for both IL and AL horses. No significant differences were recorded between horses competing at the advanced or intermediate level in the duration of the WU phases of either the Dr or $\mathrm{Cr}$ tests. In the $\mathrm{Sj}$ test, however, the mean time spent in the WU phase was significantly longer for the AL horses $(P=0.04)$. No significant difference was recorded between the IL and AL horses in the distance travelled during the WU phases for any of the tests. No significant differences were identified for maximum running speed, although during the WU phase of the $\mathrm{Sj}$ test, IL horses exhibited faster mean speeds $(P=0.05)$. With regard to the $\mathrm{C}$ phase of each test, no significant 
differences were identified for distance travelled between IL and AL horses in the Dr or Sj. During the $\mathrm{Cr}$ competition, however, the mean distance travelled was further and the duration of the test was longer in AL horses ( $P=0.01$ and $P=0.05$, respectively).

\section{Correlation between $H R$ and speed}

No correlations were identified between mean running speed and HR during the WU or C phase of the Dr test. Similarly, no correlation was identified for the WU phase of the Cr test, but running speed and $\mathrm{HR}$ were found to be highly correlated during the $\mathrm{C}$ phase $(P=0.004 \mathrm{r}=+0.78)$. Once again, no correlations were identified for the WU phase of $\mathrm{Sj}$ test, but running speed and HR were significantly correlated during for the $\mathrm{C}$ phase $(P=0.05 \mathrm{r}=-0.60)$.

Heart rate recovery $(H H R)$

The HRR data obtained for horses competing in the two ability classes (IL and AL) were pooled. For the Dr test, data were available for 9 horses. The mean percentage of HR peak at the end of the competition was $57 \pm 6 \%$, while after 180 seconds it was $37 \pm 8 \%$; the power curve appears to describe this phenomenon, even if $\mathrm{r} 2$ is very low $(0.21)$. For the $\mathrm{Sj}$ test, data were available for 8 horses; the mean percentage of HR peak at the end of the competition was $59 \pm 10 \%$, while after 180 seconds it was $41 \pm 4 \%$; a logarithmic curve was able to describe the phenomenon, but the $\mathrm{r} 2$ is once again low (0.34). For the Cr test, data were available for 7 horses. The mean percentage of HR peak at the end of the competition was $92 \pm 10 \%$, while after 180 seconds it was $53 \pm 8 \%$; a power curve appears to describe the phenomenon well, showing good correlation $(\mathrm{r} 2=0.92)$.

Lactate level

Lactate levels following the $\mathrm{C}$ phase of the Dr test were below the range of detection of the portable lactacidometer (less than $0.7 \mathrm{mmol} / \mathrm{L}$ ). The mean concentration of lactate following the $\mathrm{C}$ phase of the $\mathrm{Sj}$ test was $0.84 \pm 0.21 \mathrm{mmol} / \mathrm{L}$ in the IL horses and $1.08 \pm 0.41 \mathrm{mmol} / \mathrm{L}$ in the AL horses, but the difference between classes was not statistically significant. Following the $\mathrm{C}$ phase of the $\mathrm{Cr}$ test, lactate levels were significantly higher in AL horses than in IL horses: 9.45 \pm 4.46 vs. 3.27 \pm 3.17 , respectively $(P=0.01)$.

Theoretical energy expenditure (EE)

Statistical differences between the horse groups were only observed in $\mathrm{EE}$ for the $\mathrm{C}$ phase of the $\mathrm{Cr}$ test, with AL horses exhibiting higher levels than IL horses: $26.55 \pm 3.95$ vs. 19.01 $\pm 3.01 \mathrm{MJ}$,

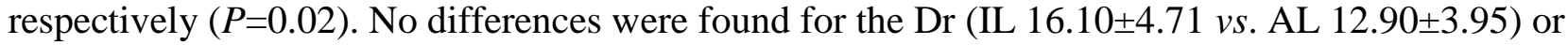

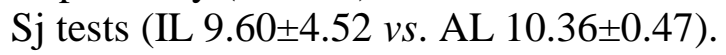

\section{Discussion}

Very few studies have investigated the physiological efforts made by horses competing in short format eventing competitions despite the relevance of quantifying workload for the optimisation of training regimens and preparation of programmes in the run-up to a competition. The present study evaluated indices of workload (HR, Lact, D, S, HRR) during the WU and C phases of each eventing test as a means to investigate whether the workload placed upon horses competing at the AL is higher compared to that placed on horses competing at the IL.

\section{Warm-up phases}

During the WU phase of the dressage test, horses competing at the IL presented higher values of $\%$ HRmean compared to those competing at the AL, even though lower levels of skill are required at the IL. The difference cannot be ascribed to the mean speed or distance travelled, since these parameters were not statistical different between the groups. This implies that other factors must have influenced the HR values. Indeed, even though the determination of HR during exercise provides an objective tool for estimating workload, for values under 120-140 bpm factors such as the level of horse excitement may influence the relationship between work intensity and HR (Dekker et al., 2007). In our study, the IL group was composed of non professional young riders and inexperienced horses, which could confound observations due to nervousness and lack of experience and may account for the large standard deviation recorded during the WU phase. The mean duration of the dressage test warm-up phase was similar to that observed by Williams et al. (2009). No differences in WU duration were found between groups (IL vs. AL) despite the 
higher difficulty level for AL horses. Although no 'optimal' warm-up protocols have been established, it is generally accepted that 20-30 minutes of warm-up are suitable for preparing horses for competition. According to Murray and co-workers (2006b), who analysed the duration of 267 warm-up sessions performed at different dressage levels, more intense warm-ups in terms of duration (including the time spent at different paces) and the design of the warm-up are positively associated with final scores. Although the total time in terms of minutes dedicated to warm-up is not sufficient alone to evaluate the quality of the warm-up, no evaluation of warm-up design in terms of gait or specific movement was performed in our study; a more in-depth study should consider the features of the exercises performed, especially taking into account the technical skills required in the AL class.

With respect to the WU phase of the show jumping test, AL horses spent more time warming-up than IL horses (32 vs. 16 min, respectively) at a statistically lower mean speed (137 vs. $170 \mathrm{~m} / \mathrm{min}$, respectively) that could account for statistical differences in \%HRmean (45.6 vs. 51.2, respectively). This finding partially agrees with that obtained by Whitaker et al. (2008). The authors observed significant differences in the total time spent warming-up between different competition levels; intermediate level participants warmed their horses up for longer periods of time than lower level competitors.

No differences were recorded between AL vs. IL groups in HR data recorded during the WU phase of the Cross country test. The duration of the warm-up was approximately 28 minutes in both groups. Much longer durations were reported by Murray et al. (2006), who recorded WU durations of 55 minutes and 42 minutes in $\mathrm{CCI} 2 *$ horses competing in long and short format cross country competitions, respectively.

Although it is not possible to define an optimal WU protocol using the indices of workload recorded in the present study, we can observe that the horses of the present investigation completed WU intensities that generally required 50\% Hrmax. The values recorded for Dr competition were between $45-42 \%$ of the HRpeak, between $51-46 \%$ for the $\mathrm{Sj}$ and between $53-54 \%$ for the $\mathrm{Cr}$ competition respectively for IL and AL.

As suggested by Tyler et al. (1996), we can conclude that warm-up intensities requiring 50\% VO2max for 5 minutes are likely to elicit an improved utilisation of aerobic energy resources during

a competition. The HR\%mean data recorded during our study indicate that the warm-up protocols used were sufficient for the effort required, especially during the cross country phase, where adequate warming up is very important for preventing fatigue that can affect the successful completion of the test.

However, an assessment that comprises only the total time dedicated to warm-up or \%HRmean is not sufficient for evaluating the quality of the warm-up, especially for the dressage test, where gait or specific movement are judged. For example, especially at the intermediate level, variables that include rider characteristics and horse age could affect optimal warm-up time, especially in relation to adapting to the competition environment and to rider stress.

Moreover, since no significant differences in time, distance or speed were observed in the show jumping competition between AL and IL, a similar workload is suggested for the two classes; for this reason, intermediate horses should be warmed-up for an appropriate time period that is similar to that of experienced horses. An appropriate WU duration would allow horses to achieve specific speeds and jumping efforts.

Considering the high workload imposed by cross country courses (characterised by the recruitment of anaerobic metabolism), WU programmes should be long enough and of the right intensity and quality to enable horses to achieve the physiological adaptations they need to perform optimally. The aim of the WU phase is to enhance blood supply to working muscles and to increase oxygen and substrate utilisation during the competition. In our opinion, the fact that AL horses exhibited higher lactate concentrations at the end of the cross country competition compared to IL horses suggests that they may require longer and more specific warm-up programmes. For this reason, 
riders should optimise warm-up duration and quality in accordance with the competition category in which they are competing.

\section{Competition phases}

As observed in the WU phase of the dressage test, horses competing at the IL exhibited higher mean heart rates during dressage test performances than those competing at the AL, despite the fact that the performance demands of IL horses were lower. In contrast, no differences were found in any parameter measured between the AL and IL during the show jumping competition; the HRmean values recorded during the $\mathrm{Sj}$ competition phase are similar to those detected by Sloet van Oldruitenborgh-Oosterbaan et al. (2006) and Marlin (2007), who studied horse workload during jumping exercises.

HRmean values recorded during the cross country competition are similar to those previously observed by Serrano et al. (2002) and Marlin (2007). No differences were found between the IL and AL groups and our data show that the cross country test provokes near-maximal heart rates and represents one of the most demanding tests of the eventing competition.

Although HRmean values were not different between groups, lactate levels were higher in AL horses. This finding suggests that the specific competition features of this class, such as the longer distance and duration have a strong influence upon this parameter.

Low levels of lactate were recorded at the end of the dressage competition in both groups, implying that the effort required was similar in both groups of horses and that exercise was entirely aerobic. Indeed, the HRmean and HR peak values recorded during the competition were below the mean anaerobic threshold limit determined in this same group of horses of $4 \mathrm{mmol} / \mathrm{L}$ (Valle, unpublished data).

No statistically significant difference in lactate levels was evident between the groups following the show jumping competition, and the values once again remained below the anaerobic threshold. According to Clayton (1991), the lactate concentration reached may be related to a horse's fitness level, the height and number of fences jumped, the length of a show jumping course and the mean speed of the performance. The low lactate increases recorded here may therefore be related to the specific features of the show jumping course of this particular competition, which resulted in little anaerobic exercise.

Changes in blood lactate levels reflect the contribution of anaerobic metabolism to energy production and are correlated with the intensity of exercise performed; indeed the increase in blood lactate can be used to assess the fitness or work capacity of horses (Murray et al., 2006). Since the anaerobic threshold was exceeded in both AL and IL horses following the cross country competition, an active recovery at trot should be recommended to promote lactate removal in these horses.

Heart rates recorded during the first three minutes following the end of competition were elaborated to obtain values of HRR. Few studies exist in the literature evaluating heart rate recovery, probably due to the difficulty in obtaining the required measurements in the field (Evans et al., 2006). Thus, further research is needed to collect more data in order to allow comparison, particularly if the aim is to identify exercise intolerance. Three minutes following the completion of the dressage and show-jumping tests, heart rates had dropped to approximately $37 \%$ and $41 \%$ of the HR peak, respectively (AL and IL pooled data). The low-intensity of exercise during the competition phases of these tests can explain the fast nature of the recovery. Analysing the dressage and show-jumping mean regression lines, we identified a weak correlation between time and recovery \%HR peak. This is probably due to the low heart rates reached during these competition tests and the 'exciting factors', including both horse and riders factors, which may influence heart rate recovery (Caola et al., 2001).

In contrast, for the cross-country phase we found that heart rates had decreased by about $53 \%$ of HR peak values 180 seconds after the completion of the course and that the mean regression line was characterised by high correlation $(\mathrm{r} 2=0.92)$. Similar findings were obtained by Bitschnau et al . (2010) in warmblood horses during a treadmill test, in which a post exercise decrease in heart rate 
to $50 \%$ of the maximal HR in the first 3 minutes was recorded. Thus, HRR is an appropriate parameter for evaluating workload after high-intensity, but not low-intensity, exercise and may represent a useful marker of fitness level, ability and soundness; however, the usefulness of this parameter should not be overestimated for the evaluation of workload following low-intensity exercise, when HR recovery may be influenced by other factors.

In this study, we also used HR and mean running speeds to calculate the theoretical energy expenditure (EE) of the horses. Coenen (2008) elaborated a model to predict energy expenditure during work, in which heart rate is considered as a biological scale for estimating exercise intensity since it responds to metabolic demands and maximum oxygen uptake (VO2max) (Coenen, 2010). However, since HR is also affected by catecholamines at values below 120-130 bpm, we also decided to use the values of running speed, as proposed by the Coenen. We therefore propose a mean theoretical value of energy expenditure derived from HR and S data, which is then converted into UFC (Horse Feed Units). Based on the horse's net energy (NE) system that was proposed and first introduced in France (Martin Rosset, 2012), it is possible to calculate an energy cost per hour of work done by a sport horse according to the nature and duration of exercise. Horse work is classified as light, medium or intense, and the additional energy requirements per hour are $14 \mathrm{MJ} / \mathrm{h}$ for light work, 19-24 MJ/h for medium work, 25-33 UFC/h for intense work and 34-42 MJ/h for very intense work.

By comparing the values of EE calculated for the two groups in relation to the cross country test, a significantly higher EE was observed for the horses competing at the AL, confirmed by the higher workloads placed upon this category of horse. The Cr test is the most demanding of the eventing competition and differences in the efforts required to complete the test, in terms of EE and lactate production, exist between competition categories, reflecting the differences in course design at the different levels (e.g. duration, distance and size of jumps)

Moreover, since dressage and show-jumping tests were performed on the same day of the competition (day 1), it is necessary to sum the theoretical EEs for both tests; this leads to a value of energy expenditure in the 'intense work' range for the first day of competition as well as for the second day when the cross country test is performed. Energy supply is very important in sport horses (Dekker et al., 2007) and although the estimation of EE by speed or HR is not precise, it provides the most objective and easy tool for estimating workload in horses.

\section{Conclusions}

On the basis of our results, we can confirm the cross-country test to be the most demanding in the short format eventing competition. A comparison of the intermediate and advanced level competition classes revealed that greater levels of muscular and energetic effort in terms of lactic acid production and energy expenditures are required in the advanced class. Moreover, due to the greater distances covered, the greater fence heights and the presence of hills in the AL tests, an active recovery at the trot should be recommended following the completion of the cross country tests to favour the removal of built up lactate. Our results also indicate that training programmes that stimulate adaptation to anaerobic metabolism should be considered; as should and the potential benefits of administrating a supplement to help remove the lactate produced during the cross country competition, especially for horses performing at the advanced level. Horses should also receive adequate amounts of feed to restore energy balance. The calculation of heart rate recovery is an appropriate parameter for gauging workload following high-intensity exercise, but after lowintensity work it is more likely to be influenced by external factors. Since warm-up sessions allow the body to achieve physiological adaptation to the subsequent exercise, riders should optimise horse warm-up durations and quality depending on their competition category, although additional research still needs to be performed in this area to identify optimal warm-up programmes. The study of workload in other classical equestrian disciplines like dressage and show jumping is also important so that the workload demand placed 
on horses in different disciplines can be compared.

\section{References}

Bitschnau, C., Wiestner, T., Trachsel, D.S., Auer, J.A., Weishaupt, M.A., 2010. Performance parameters and post exercise heart rate recovery in Warmblood sports horses of different performance levels. Equine Veterinary Journal 42: 17-22.

Caola, G., Piccione, G., 2001. Aggiustamenti all'esercizio fisico e adattamenti all'allenanento dell'apparato cardiocircolatorio del cavallo. In Caola, G. (ed.) Fisiologia dell'Esercizio Fisico del Cavallo. Calderoni ed agricole, Bologna pp 71-86.

Clayton, H.M., 1991. Conditioning Sport Horses. Sport horse publications, Canada.

Coenen M., 2008. The siutability of heart rate in the prediction of oxygen consumption, energy expenditure and energy requirement of the exercising horse. In: Nutrition of the excercizing horse. EAAP series 125. Wageningen Academic Publishers, the Netherlands 139-146.

Coenen M., 2010. Remarks on the benefits of heart rate recordings. In proceeding of the Kentucky equine research conference. Lexington, KY 42-54.

Dekker, H., Marlin, D., Alexander, L., Bishop, R., Harris, P., 2007. A pilot study investigating the relationship between perceived and actual workload and estimated energy intake in riding centre horses. Equine and Comparative Exercise Physiology 4: 7-14.

Evans, D.L., Jeffcott, L.B., Knight, P.K., 2006. Performance-related problems and exercise physiology. In: Higgins, A.J. Snyder J.R. (ed.), The Equine Manual (Second Edition).

Elsevier Saunders, 1059-1104.

Gramkow H.L., Evans D.L., 2006. Correlation of race earnings with velocity at maximal heart rate during a field exercise test in thoroughbred racehorses. Equine Veterinary Journal Supplement 36, 118-122..

Henneke D.R., Potter G.D., J.L. Kreider J.L.,. Yeates B.F, 1983. Relationship between condition score, physical measurements and body fat percentage in mares. Equine Veterinary Journal 15: 371-372

Marlin, D.J., 2007. Exercise Physiology of Eventing. Available at:

http://www.davidmarlin.co.uk/PDFs/Demands\%20of\%20eventing\%20MARLIN\%20v4b.pdf

Martin Rosset W., 2012. Nutrition et alimentation des chevaux. Editions Quae, Versailles

Cedex, Fr.

Muñoz, A., Riber C., Santisteban, R., Rubio, M.D., Agüera, E.I., Castejón, F.M., 1999.

Cardiovascular and metabolic adaptations in horses competing in cross country events.

Journal of Veterinary Medical Science, 61: 13-20.

Murray, J.K., Senior, J.M., Singer, E.R., 2006. A comparison of cross-country recovery rates at CCI $2 *$ with and without steeplechase competitions. Equine Veterinary Journal Supplement

36:133-138.

Murray, R.C., Manna, S., Parkin, T.D.H., 2006 b. Warm-up in dressage competitions:

association with level, competition type and final score. Equine and Comparative Exercise Physiology, 3: 185-190.

Reis V., M., Van den Tillaar R., Marques M.,C., 2011. Higher precision of heart rate compared with VO2 to predict exercise intensity in endurance-trained runners. Journal of Sports Science and Medicine 10: 164-168.

Rogers, C.W., Rivero J.L.L., Band van Lindner, A., Sloet van Oldruitenborgh-Oosterbaan, M..M., 2007. Describing workload and scientific information on conditioning horses. Equine and Comparative Exercise Physiology 1: 1-6.

Serrano, M., Evans, D., Hodgson, J., 2002. Heart rate and blood lactate responses during exercise in preparation for eventing competition. Equine Veterinary Journal 30: 135-139. Sloet van Oldruitenborgh-Oosterbaan, M.M., Spierenburg, A.J., van den Broek, E.T.W., 2006. The workload of riding-school horses during jumping. Equine Veterinary Journal 36: 93-97. Sloet van Oldruitenborgh-Oosterbaan, M.M., van den Broek, E.T.W., Spierenburg, A.J., 2008. Evaluation of the usefulness of the portable device Lactate Pro for measurement of lactate 
concentrations in equine whole blood. Journal of Veterinary Diagnostic Investigation 20: 8385 .

Tyler C.M., Hodgson D.R., Rose R.J.,1996. Effect of a warm-up on energy supply during high intensity exercise in horses. Equine Veterinary Journal 28:117-120.

Whitaker, T.C., Mills, A., Duxbury, L.J., 2008. Horse warm-up regimes at two different competitive levels of show jumping: a pilot study. Comparative Exercise Physiology 5: 105106.

Williams, R.J., Chandlera, R.E., Marlin, D.J., 2009. Heart rates of horses during competitive dressage. Comparative Exercise Physiology 6: 7-15.

Table 1. Description of the intermediate level and advanced level eventing competition

\begin{tabular}{ccc}
\hline & Intermediate level $(\mathbf{n = 5})$ & Advanced level $(\mathbf{n = 5})$ \\
\hline Dressage & $\begin{array}{c}\text { Marks given for medium } \\
\text { walk, working and medium } \\
\text { trot and working canter }\end{array}$ & $\begin{array}{c}\text { Marks given for medium and extended } \\
\text { walk, working, medium and collected } \\
\text { trot, working, medium and collected } \\
\text { canter, rein back, and shoulder in at trot. }\end{array}$ \\
Show jumping & Distance $390 \mathrm{~m}$ & $\begin{array}{c}\text { Distance } 400 \mathrm{~m} \\
\text { Speed } 350 / \mathrm{min}\end{array}$ \\
& Time $67 \mathrm{~s}$ & Time $69 \mathrm{~s}$ \\
Fence height $1.10 \mathrm{~m}$ & Fence height $1.15 / 1.20 \mathrm{~m}$ \\
Cross country & Distance $2460 \mathrm{~m}$ & Distance $2700-3100 \mathrm{~m}$ \\
& Speed $510 \mathrm{~m} / \mathrm{min}$ & Speed $520-530 \mathrm{~m} / \mathrm{min}$ \\
Time $4: 50 \mathrm{~min}$ & Time $5: 20-5: 51 \mathrm{~min}$ \\
& Fence height $1.05 \mathrm{~m}$ & Fence height $1.10-1.15 \mathrm{~m}$ \\
\hline
\end{tabular}

Table 2. The age ranges of horses competing in the two ability categories and the range of maximum HR (HRpeak, bpm) recorded during a fast gallop training session.

\begin{tabular}{ccc}
\hline & $\begin{array}{c}\text { Age, years } \\
\text { (min-max range) }\end{array}$ & $\begin{array}{c}\text { HRpeak } \\
\text { (range, bpm) }\end{array}$ \\
\hline Advanced level & $9-13$ & $200-213$ \\
Intermediate level & $6-13$ & $190-228$ \\
\hline
\end{tabular}


Table 3. Heart rate values recorded during the warm-up and competition phases of each test of the eventing competition in intermediate level (IL) and advanced level (AL) horses (means plus standard deviations, SD)

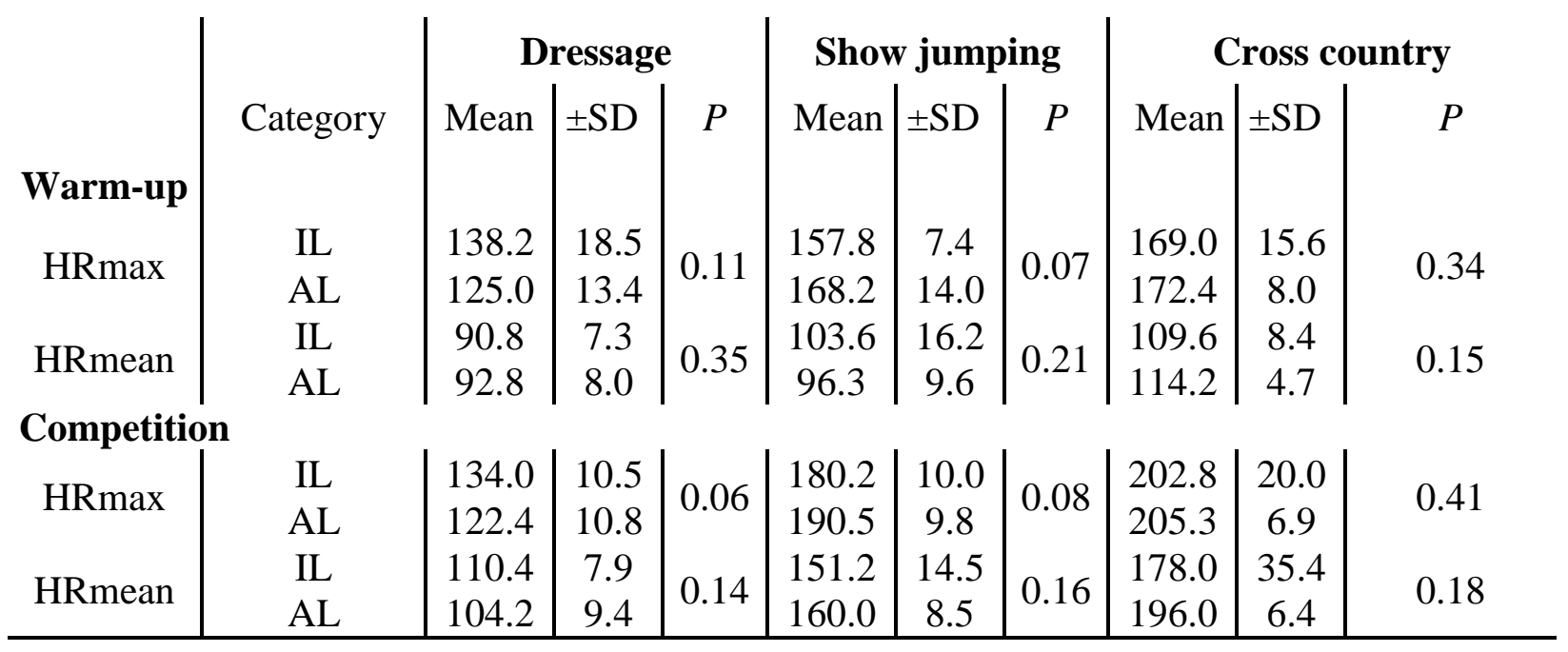

Table 4. Heart rates recorded during the warm-up and competition phases of each test of the eventing competition expressed as a percentage of HR peak obtained during the fast gallop test (\%HRmean) and maximum HR (\%HRmax) in intermediate level horses (IL) and advanced level horses (AL) (means plus standard deviations, SD)

\begin{tabular}{|c|c|c|c|c|c|c|c|c|c|c|}
\hline \multirow[b]{2}{*}{ Warm-up } & \multirow[b]{2}{*}{ Category } & \multicolumn{2}{|c|}{ Dressage } & \multicolumn{4}{|c|}{ Show jumping } & \multicolumn{3}{|c|}{ Cross country } \\
\hline & & Mean & $\pm \mathrm{SD}$ & $P$ & Mean & $\pm \mathrm{SD}$ & $P$ & Mean & $\pm \mathrm{SD}$ & $P$ \\
\hline \multirow{2}{*}{$\%$ HRmax } & IL & 68.6 & 10.8 & \multirow{2}{*}{0.05} & 77.6 & 7.2 & \multirow{2}{*}{0.36} & 83.0 & 3.9 & \multirow{2}{*}{0.13} \\
\hline & AL & 58.0 & 5.9 & & 79.2 & 5.9 & & 80.6 & 2.2 & \\
\hline \multirow[t]{2}{*}{$\%$ HRmean } & IL & 45.4 & 2.5 & \multirow{2}{*}{0.04} & 51.2 & 6.3 & \multirow{2}{*}{0.05} & 52.6 & 3.9 & \multirow{2}{*}{0.21} \\
\hline & $\mathrm{AL}$ & 42.2 & 2.6 & & 45.6 & 2.4 & & 54.2 & 1.8 & \\
\hline \multicolumn{11}{|c|}{ Competition } \\
\hline \multirow{2}{*}{ \%HRmax } & IL & 65.8 & 2.8 & \multirow{2}{*}{$<0.01$} & 88.6 & 8.2 & \multirow{2}{*}{0.38} & 96.2 & 3.9 & \multirow{2}{*}{0.09} \\
\hline & $\mathrm{AL}$ & 57.2 & 3.6 & & 89.8 & 1.5 & & 99.0 & 1.0 & \\
\hline \multirow[t]{2}{*}{$\%$ HRmean } & IL & 54.4 & 4.3 & \multirow{2}{*}{0.01} & 73.0 & 6.0 & \multirow{2}{*}{0.12} & 83.4 & 11.3 & \multirow{2}{*}{0.06} \\
\hline & $\mathrm{AL}$ & 48.2 & 1.9 & & 76.6 & 2.1 & & 93.6 & 2.3 & \\
\hline
\end{tabular}


Table 5. Duration, distance travelled, mean and maximum running speed during warm-up and competition phases of each test in advanced (AL) and intermediate level (IL) horses (mean and standard deviation, SD) (time durations were rounded to the nearest minute)

\begin{tabular}{c|c|c|c|c|c|c|c|c|c|c}
\hline Warm-up & \multicolumn{9}{|c|}{ Dressage } & \multicolumn{2}{|c|}{ Show jumping } & \multicolumn{3}{|c}{ Cross country } \\
& Category & Mean & \pm SD & P & Mean & \pm SD & P & Mean & \pm SD & $P$ \\
Duration & IL & 38 & 20 & & 16 & 9 & & 28 & 8 & \\
(min) & AL & 35 & 13 & 0.34 & 32 & 14 & 0.04 & 28 & 3 & 0.46 \\
Distance, & IL & 4140 & 2050 & & 2150 & 950 & & 4130 & 1200 & \\
$(\mathrm{~m})$ & AL & 4280 & 1510 & 0.45 & 3550 & 1830 & 0.11 & 4400 & 430 & 0.20 \\
S Mean & IL & 134 & 19 & & 170 & 33 & & 119 & 64 & \\
$(\mathrm{~m} / \mathrm{min})$ & AL & 134 & 19 & 0.50 & 137 & 9 & 0.05 & 170 & 1 & 0.07 \\
Smax & IL & 258 & 36 & & 380 & 70 & & 379 & 117 & 0.19 \\
$(\mathrm{~m} / \mathrm{min})$ & AL & 248 & 42 & 0.35 & 321 & 38 & 0.10 & 433 & 43 &
\end{tabular}

\section{Competition}

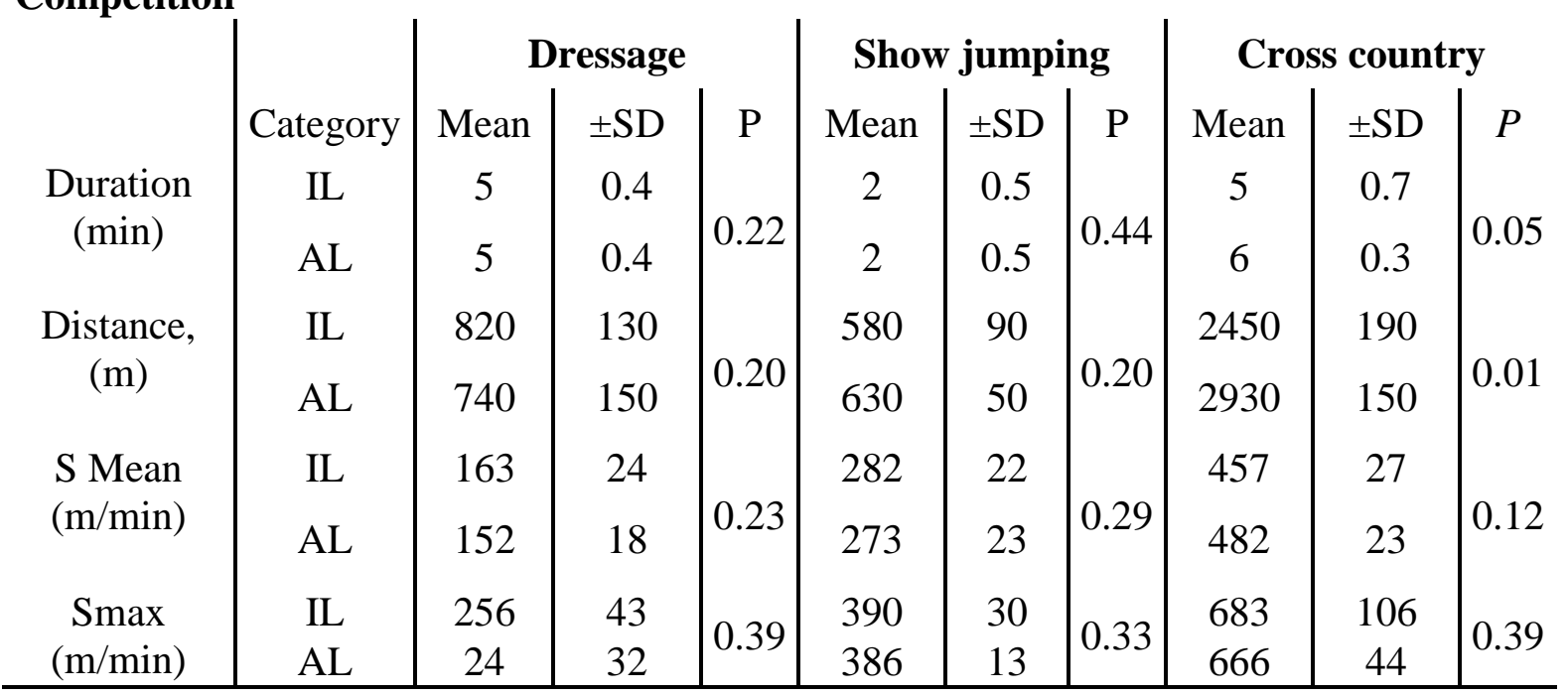

